\title{
The possible explaining of some controversial effects by a vortexial atom model
}

\begin{abstract}
In the paper is presented a vortexial pre-quantum model of atom, based on a vortexial type of electron' and proton' magnetic moment, resulted in a cold genesis theory(CGT) as etherono-quantonic vortex $\Gamma_{\mu}^{*}(\mathrm{r})=\Gamma_{\mu}\left(\mathrm{r}_{\mu}{ }^{\prime}\right)+\Gamma_{\mathrm{B}}\left(\mathrm{r}>\mathrm{r}_{\mu}{ }^{\prime}\right)$, of heavy' tachyonic etherons $\left(\mathrm{m}_{\mathrm{s}} \approx 10^{-60} \mathrm{~kg} ; \mathrm{w}>\mathrm{c}\right)$ - generating the magnetic potential $\mathrm{A}$, and of quantons $\left(\mathrm{m}_{\mathrm{h}}=\mathrm{h} \cdot 1 /\right.$ $\mathrm{c}^{2}=7.37 \times 10^{-51} \mathrm{~kg}$ )-generating vortex-tubes $\xi_{B}$ that materializes the B-field lines of the magnetic induction, the proton's magnetic moment resulting by a degenerate Compton radius. The model may explain the 'hydrino' atom, with $n=1 / 2$, the tachyonic speed of the electronic neutrino and the Kervran effect of biological nuclear transmutations. By the multi-vortexial model of nucleon resulted in CGT, are explained also some astrophysical observation which sustains the CGT's hypothesis of pulsatile antigravitic pseudo-charge and gravitational waves generating at the surface of a 'black hole type star by matter $\rightarrow$ energy conversion, resulting also the possibility of cosmic dust's cold forming.
\end{abstract}

Keywords: vortexial atom, hydrino, OPERA experiment, Kervran effect, antigravitic charge, gravitational waves
Volume 4 Issue 2 - 2020

\section{Marius Arghirescu}

State Office for Inventions and Trademarks, Patents Department, Romania

Correspondence: Marius Arghirescu, State Office for Inventions and Trademarks, Patents Department; Romania, Emailmaris3a@yahoo.com

Received: April 23, 2020 | Published: April 30, 2020

\section{Introduction}

In a Cold Genesis Theory of Matter and Fields of the author, (CGT${ }^{1,2}$ ), based on the Galilean relativity the discovered elementary particles are explained by a vortex model, of composite fermion type, as Bose Einstein Condensate of $\mathrm{N}^{\mathrm{p}}$ gammons considered as thermalized pairs: $\gamma^{*}=\left(\mathrm{e}^{-} \mathrm{e}^{+}\right)$of axially coupled electrons with opposed charges which became degenerate electrons inside the neutral $\mathrm{N}^{\mathrm{p}}$ cluster, i.e- quasielectrons with diminished mass, charge and magnetic moment, i.e: $\mathrm{m}_{\mathrm{e}}^{*} \approx 0.81 \mathrm{~m}_{\mathrm{e}} ; \mathrm{e}^{*} \approx(2 / 3) \mathrm{e}$;

$$
\mu_{\mathrm{e}}^{*} \approx \mu_{\mathrm{e}}\left(2.79 \mathrm{~m}_{\mathrm{e}} / m_{\mathrm{P}}\right) \approx \mu_{\mathrm{P}, 1,2}
$$

The particle's magnetic moment ${ }_{\mathrm{e}}^{*}$ results in CGT as etheronoquantonic vortex: $\Gamma_{\mu}^{*}(\mathrm{r})=\Gamma_{\mathrm{A}}+\Gamma_{\mathrm{B}}$, of heavy (,sinergonic”) etherons $\left(\mathrm{m}_{\mathrm{s}} \approx 10^{-60} \mathrm{~kg}\right)$ generating the magnetic potential $\mathbf{A}$ and of quantons $\left(\mathrm{m}_{\mathrm{h}}=\mathrm{h} \cdot 1 / \mathrm{c}^{2}=7.37 \times 10^{-51} \mathrm{~kg}\right)$ - generating vortex-tubes $\xi_{\mathbf{B}}$ that materializes the $\mathbf{B}$-field lines of the magnetic induction.

The superposition of the $\left(\mathrm{N}^{\mathrm{p}}+1\right)$ quantonic vortices: $\Gamma_{\mu}^{*}$ of the protonicquasielectrons generates inside a volume with the radius: $r^{a}{ }_{\mu}=2.35 \mathrm{fm}^{1-4}$ a total dynamic pressure: $P_{n}=\left({ }^{1 /}{ }_{2}\right) \rho_{n}(r) \cdot c^{2}$ which gives a nuclear potential: $\mathrm{V}_{\mathrm{n}}(\mathrm{r})$, in an eulerian form, having a variation according to eqn:

$$
\begin{gathered}
V_{n}(r)=u_{i} P_{n}(r)=V_{n}^{0} \cdot e^{-r / \eta^{*}} ; V_{n}^{0}=u_{i} P_{n}^{0}=\left({ }_{i}^{u}{ }_{i}\right) r_{n}{ }^{0} \times c^{2} ; \\
\text { with }: \eta^{*}=0.8 \mathrm{fm}[2],
\end{gathered}
$$

$\left(v_{\mathrm{i}}(0.6 \mathrm{fm}) \approx 0.9 \mathrm{fm}^{3}-\right.$ the nucleon's impenetrable quantum volume of nuclear interaction). Also, the neutron results in CGT by a specific "dynamid" model, with a degenerate electron with degenerate magnetic moment: $\mu_{\mathrm{e}}^{\mathrm{s}} \approx-4.6 \mu_{n}\left(\mu_{n}\right.$ - the nuclear magneton) rotated inside the quantum volume of a proton by the etherono quantonic vortex $\Gamma_{\mathrm{P}}$ of the protonic magnetic moment $\mu_{p}$, with a speed $\mathrm{v} \approx 1.7 \times 10^{-2} \mathrm{c}$, to an orbital with a radius: $r_{e}^{*} \approx 1.28 \mathrm{fm}$, under dynamic equilibrium of forces on tangent and radial directions. ${ }^{1,2}$

The nuclear fission of the nucleus, particularly- the superasymmetric nuclear fission, ${ }^{3}$ may be explained in CGT by a deuteronic self-resonance mechanism which generates a decrease in the value of the interaction potential $\mathrm{V}_{\mathrm{n}}$ between nucleons by nucleonic vibrations also in a quasi-crystal nucleus, ${ }^{1,2}$ in portions with incompleteness of the quasi-crystalline nucleonic network or with excedentary nucleons, which leads to a higher 'vibration liberty' $1_{v}$ of these weakly bound nucleons and this vibration decreases the value of the scalar nucleonic potential and determines either the nucleus fission into sub-nuclei with symmetrical quasi-crystalline forms (often - "magical" or quasi-stable forms), or gamma vibration spectra, as a result of the self-resonance of nucleons less strongly bound to the nucleus, according to a spindependent nuclear potential relation $\left(\mathrm{CGT},{ }^{1,2}\right)$ :

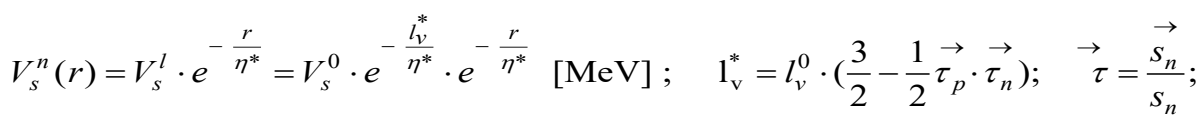


in which: $\mathrm{V}_{\mathrm{s}}^{0}=109.8 \mathrm{MeV} ; \eta^{*}=0.8 \mathrm{fm},{ }^{4} 1_{\mathrm{v}} \cong \mathrm{A}_{\mathrm{v}}$, (the vibration amplitude $) ; 1_{\mathrm{v}}^{0}\left(\mathrm{E}_{\mathrm{v}}^{\mathrm{s}}\right) \cong 1 \mathrm{fm}$-for the deuteron;

$$
\left(1_{\mathrm{v}}\left(\mathrm{E}_{\mathrm{v}}=0\right)=0\right) ; 1_{\mathrm{v}} \sim \mathrm{E}_{\mathrm{v}}=1 / 2 \mathrm{~m}_{\mathrm{n}} \mathrm{v}_{\mathrm{n}}^{2}=1 / 2 \mathrm{k}_{\mathrm{v}} \cdot \mathrm{A}_{\mathrm{v}}^{2} .
$$

The relation (6) corresponds also to the generalized nuclear mode by the conclusion that the vibration energy of the nucleons $\in_{\mathrm{v}} \sim \mathrm{r}^{2}$ decreases the value of nuclear potential well: $\mathrm{V}_{\mathrm{s}}^{1}$. The degeneration of the nucleonic potential through the nucleon's vibration, according to eqn. (6), explains also the transformation mechanism of the compound nucleus by interaction with low energy particles up to $2 \mathrm{MeV}$, as in the case of the $\mathrm{Be} 9$ nucleus that can be transformed with a gamma quantum of only $1.78 \mathrm{MeV}$, even if the binding energy given by the sum of its nucleons is $\sim 58 \mathrm{MeV}$.

\section{The atomic electrons rotating cause}

In CGT, the atom results phenomenologically as formed mainly at cold, as quantum-vortexial system, the electrons being circulated with

$$
\rho_{\mathrm{s}}{ }^{\prime}(\mathrm{r}) \times\left(\mathrm{w}-\mathrm{v}_{\mathrm{e}}\right)^{2}=\rho_{\mathrm{R}}(\mathrm{r}) \times \mathrm{v}_{\mathrm{e}}{ }^{2}(\mathrm{r}) ; \quad\left(\rho_{\mathrm{s}}(\mathrm{r})=\rho_{\mathrm{s}}{ }^{\mathrm{a}} \times(\mathrm{a} / \mathrm{r})^{2} ;(\mathrm{c}<\mathrm{w} \leq \sqrt{ } 2 \mathrm{c})\right.
$$

The electron $\mathrm{v}_{\mathrm{e}}(\mathrm{r})$ - speed variation in the hydrogen atom results from the quantification law of the orbital kinetic moment of electron:

$$
\mathrm{v}_{e}(r)=c \cdot \sqrt{\frac{2 a}{r}} ; \quad \frac{\mathrm{v}_{\mathrm{o}}}{c}=\sqrt{\frac{2 a}{r_{0}}}=\frac{1}{137}=\alpha ; \quad r^{0}=0,53 \stackrel{\circ}{A}
$$

For $\mathrm{r}>>\mathrm{a},\left(\mathrm{w}-\mathrm{v}_{\mathrm{e}}\right) \approx \mathrm{w}$, so it results that:

$$
\rho_{\mathrm{R}}(\mathrm{r})=\rho_{\mathrm{s}}^{\mathrm{a}} \times(\mathrm{a} / 2 \mathrm{r}) .
$$

The eq. (5) shows also that at the distance $\mathrm{r}_{\mu}{ }^{a} \cong 2 \mathrm{a}$ from the proton, the electron is revolved by the proton's $\Gamma_{\mu}{ }^{{ }-}$- vortex (mainly-by the $\Gamma_{\mathrm{A}}{ }^{{ }^{2}}$ vortex) with the speed: $\mathrm{v}_{\mathrm{e}}{ }^{\mathrm{M}} \rightarrow \mathrm{c}$, which may be explained- in our

model, if the proton's $\Gamma_{\mu}{ }^{\mathrm{p}}$ - quantonic vortex satisfies the condition:

$$
\mathrm{r}_{\mu}{ }^{\mathrm{a}} \rightarrow 2 \mathrm{a} \Rightarrow \Gamma_{\mu}^{\mathrm{p}}\left(\mathrm{r}_{\mu}^{\mathrm{a}}\right) \rightarrow 2 \pi \mathrm{r}_{\mu}{ }^{\mathrm{a}} \mathrm{c},
$$

So the eq. (5) may be approximated by eq. (6), for $\mathrm{w} \cong \sqrt{2} \cdot \mathrm{c}$ and: $\rho_{\mathrm{s}}{ }^{\prime}(\mathrm{r}) \cong 1 / 2 . \rho_{\mathrm{s}}(\mathrm{r})$, by the semi-empiric form:

$$
\rho_{R}(r)=\frac{\rho_{s}^{a}}{2}\left(\frac{a}{r}\right)^{2} \cdot\left(\gamma \sqrt{\frac{r}{a}}-1\right)^{2} ; \Rightarrow \rho_{\mathrm{R}}(2 a) \approx \frac{\rho_{s}^{a}}{8} \cdot(\gamma \sqrt{2}-1)^{2} ; \quad \rho_{\mathrm{R}}(r) \cdot \mathrm{v}_{\mathrm{e}}^{2} \cong \frac{\rho_{s}^{a}}{2}\left(\frac{a}{r}\right)^{2} \cdot\left(\sqrt{2} c-\mathrm{v}_{\mathrm{e}}\right)^{2} ; \gamma=\mathrm{e}^{\frac{\mathrm{r}^{0}}{\mathrm{r}}}
$$

With $\gamma=\mathrm{e}^{\mathrm{r} 0 / \mathrm{r}} \rightarrow 1$. An argument for the eq. (7b) is the fact that- at $\beta$ disintegration of the neutron, the released electron has an energy corresponding to a speed close to the light speed, $\left(\mathrm{v}_{\beta}=\mathrm{k} \cdot \mathrm{c} \cong 0.92 \mathrm{c}\right)$ explained with eq. (7) by the conclusion that this speed is given to the electron of $\beta^{-}$radiation by the $\Gamma_{\mu}^{p}$ vortex of the remained proton. Also, the same vortex gives the neutrino speed. So, the atom properties may be explained by a vortexial model, different from the classic (vortexial) model proposed by Thomson and Kelvin. The apparent contradiction between the value $r_{\mu}^{\mathrm{p}} \rightarrow 2 \mathrm{a}$ and the radius: $r_{\mu}{ }^{\mathrm{p}}=0,59 \mathrm{fm}$ of the proton's $\mu_{\mathrm{p}}-$ magnetic moment, may be explained in the model by the fact that the protonic $\Gamma_{\mu}{ }^{\mathrm{p}}$ - vortex, given by its positron, generates also the $\Gamma_{w}$ - vortex of parallel polarized $\mathrm{m}_{\mathrm{w}}{ }^{*}$-vexons of proton surface, giving the $\mathrm{e}^{+}$-charge and having the confined vortexial energy: $\mathrm{w}_{\mathrm{w}}=\mathrm{w}_{\mathrm{u}}=1 / 2 \Sigma \mathrm{m}_{\mathrm{h}}\left(\omega_{\mathrm{h}} \mathrm{r}\right)^{2}=1 / 2 \mathrm{~m}_{\mathrm{w}}{ }^{*} \mathrm{c}^{2}$ contained by a chiral soliton with radius: $\mathrm{r}_{\mathrm{w}}{ }^{\mathrm{n}} \rightarrow\left(\mathrm{a}_{\mathrm{n}}-\mathrm{r}_{\mu}{ }^{\mathrm{p}}\right)$. This $\Sigma\left(\mathrm{w}_{\mathrm{w}}\right)$-vortexial energy decreases exponentially, in the proton case, and gives the value $r_{\mu}{ }^{a}$ of $\Gamma\left(\mu_{\mathrm{p}}\right)$ - proton vortex radius. Because - for the electron CF - model case, the vexons of electron surface has a degenerate Compton radius approximate equal with the electron Compton radius: $\mathrm{r}_{\mathrm{w}}{ }^{\mathrm{e}} \cong \mathrm{r}_{\mu} \mathrm{e}$, explaining the electron prequantum spin: $\mathrm{S}_{\mathrm{e}}=1 / 2 \hbar$, it results by eq. (5) that for a vexon of the proton's surface $(\mathrm{r} \cong 1.4 \mathrm{fm})$, we have for a $\boldsymbol{\Gamma}_{\mathrm{w}}{ }^{-}$ vortex: $r_{w}{ }_{w} \cong\left(r_{\mu}{ }^{e} / 1836\right) \cdot e^{1.4 / 0.93}=0.946 \mathrm{fm}$. So we may consider in eq. (7) the value: $r_{\mu}{ }_{\mu} \approx a+r_{w}{ }^{n} \cong 2.35 \mathrm{fm}$, for which: ${ }_{\mu}{ }^{p} \cong 2 \pi r_{\mu}{ }^{a}$ c. It results- in this case, a semi-empiric relation for the variation of the quantons' tangent $\mathrm{v}_{\mathrm{ct}}$-speed in the proton's $\Gamma_{\mu}{ }^{\mathrm{p}}$-vortex, which corresponds to the eqns. (4)-(7), in the form:

$$
\mathrm{V}_{\mathrm{a}}(r)=\left\{\begin{array}{l}
c, \text { for }: r<r_{\mu}^{a}=a+r_{w}^{n} \cong 2.3 \mathrm{fn} ; \mathrm{a}=1.41 \mathrm{fm} \\
c\left(\frac{r_{\mu}^{p}}{r}\right)^{\left.1-\frac{r_{\mu}^{a}}{r}\right)}, \text { for }: r \geq r_{\mu}^{a} \cong 2.3 \mathrm{fn} ; r_{\mu}^{p}=0.59 \text { or } \cong 0.6
\end{array}\right.
$$

The equality between eqs. (7a) and (7b) results for $\mathrm{r}_{\mu}{ }^{a} \cong 2.35 \mathrm{fm}$ and $\mathrm{v}_{\mathrm{e}}=\mathrm{k} \cdot \mathrm{c}=1 \mathrm{c}$, by a value $\gamma=\mathrm{e}^{\mathrm{r} 0 / \mathrm{r}}=1.095$, corresponding to: $\mathrm{r}^{0}=0.21 \mathrm{fm}$ $=\mathrm{r}^{0}{ }_{\mathrm{i}}$. 
The exponential form of $\gamma$ is given by the density of the superposed secondary $\boldsymbol{\Gamma}_{\mathrm{w}}$-vortexes in the volume of radius: $\mathrm{a}<\mathrm{r} \leq 2 \mathrm{a}$.

The quantification of the electron's kinetic moment results according to the known relation:

$$
\mathrm{L}(\mathrm{n})=\mathrm{m}_{\mathrm{e}} \mathrm{v}_{\mathrm{e}} \mathrm{r}_{\mathrm{e}}=\mathrm{n} \cdot \mathrm{h} / 2 \pi ; \mathrm{v}_{\mathrm{e}}(\mathrm{n})=\mathrm{v}_{0} / \mathrm{n}
$$

\section{The explaining of some controversial phenomena}

In accordance with the resulted relation: $\mathrm{k} \cdot \gamma \cong \sqrt{ }(2 \mathrm{a} / \mathrm{r})$, by eqs. (7a) and (7b) it results also, for $\mathrm{r} \rightarrow \mathrm{a}$, that a nuclear particle such as an emitted $\gamma$-quantum or a neutrino emitted in a $\beta$-transformation or in a mesonic transformation $\left(\pi^{ \pm} \rightarrow \mu^{ \pm}+v_{\mu}\right)$, may be accelerated by the protonic $\Gamma_{\mathrm{A}}$-vortex in a time of $\sim 10^{-23} \mathrm{~s}$ to a speed $\mathrm{v}_{\mathrm{v}}=\mathrm{k} \cdot \mathrm{c}$ with $\mathrm{k}>1$, (exceeding the light speed, c). For example, for $\mathrm{r}=1.5 \mathrm{fm}, \mathrm{k}=1.19$. So, it is possible to explain by the theory, the result of the recent OPERA experiment ${ }^{5}$ in which was observed neutrins with a speed exceeding the light speed, emitted from a CERN's accelerator and detected to the Gran Sasso lab of Italy, ("Nature", 22 sept. 2011). Is explained also the recoilless $\gamma$ - radiation emission/absorbtion phenomenon, (the Mössbauer effect). In the sametime, the value of $\rho_{\mathrm{R}}(\mathrm{r})$ for $\mathrm{r} \rightarrow \mathrm{r}_{\mu}$, explains "the stopped light" experiment (L.V.Hau, 2001) which evidenced the possibility to reduce the speed of a light beam which is passed by a small cloud of ultracold atoms of sodium forming a B-E condensate. ${ }^{6}$ The resulted pre-quantum soliton model of atom, of $\mathrm{T} \rightarrow 0 \mathrm{~K}$, which degenerates in the Bohr-Sommerfeld's model at $\mathrm{T}>0 \mathrm{~K}$, is also consistent with some other soliton models of atom [63] and allow the explaining of the electron transition on sub-fundamental level $(n=1 / 2)$ in the hydrogen atom, (i.e: the "hydrino" atom $\left.{ }^{7}\right)$ observed in some experiments of cold nuclear fusion ${ }^{7}$ by the conclusion that the quantification of the electron number of an atomic energy level: N(n), corresponds to a superficial charge density $\sigma_{e}$ of constant value for an energetic layer considered as having quasi-cylinder (barrel-like) form, of 1- height and quantified $\mathrm{r}_{\mathrm{e}}$ - radius, (Figure 1):

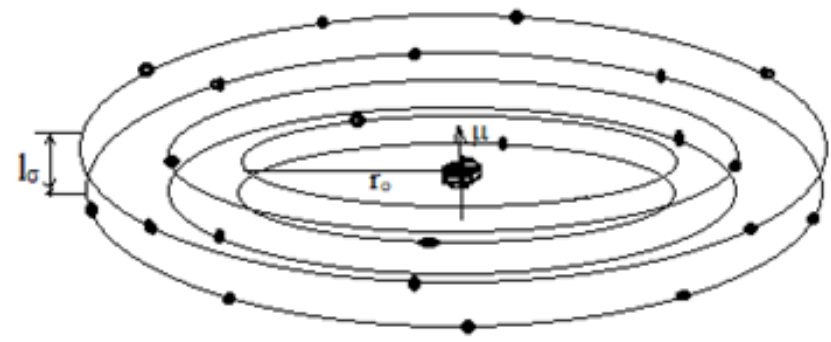

Figure I Pre-quantum atom.

$$
\begin{aligned}
& \sigma_{\mathrm{e}}=\mathrm{e} \cdot \mathrm{N}(\mathrm{n}) / 2 \pi \cdot \mathrm{r}_{\mathrm{e}} \cdot \mathrm{l}_{\mathrm{s}}=\text { const; } \Rightarrow \mathrm{N}(\mathrm{n}) \sim \mathrm{r}_{\mathrm{e}}=\mathrm{n}^{2} \cdot \mathrm{r}_{\mathrm{o}} \\
& \Rightarrow \mathrm{N}(\mathrm{n})=\mathrm{Q}(\mathrm{n}) / \mathrm{e}=\left(\sigma_{\mathrm{e}} \cdot 2 \pi \mathrm{r}_{\mathrm{e}} 1_{\mathrm{s}}\right) / \mathrm{e}=2 \mathrm{n}^{2} ;(10) \\
& \mathrm{Q}(1)=2 \mathrm{e}, \mathrm{r}_{\mathrm{o}}=\mathrm{e} /\left(\sigma_{\mathrm{e}} \cdot \pi \cdot 1_{\mathrm{s}}\right) ;\left(\mathrm{r}_{\mathrm{e}}=\mathrm{n} \cdot \hbar / \mathrm{m}_{\mathrm{e}} \mathrm{v}_{\mathrm{e}}=\mathrm{n}^{2} \cdot \mathrm{r}_{\mathrm{o}}\right)
\end{aligned}
$$

According to the model, the transition on sub-fundamental level $(\mathrm{n}=1 / 2)$ is specific to the hydrogen atom, (to the "hydrino" atom) by the condition $\mathrm{Q}(\mathrm{n}=1 / 2)=\mathrm{e}$, ( $\mathrm{H}$-atom having a single electron), condition which gives by eqn. (10) a radius for the under-fundamental level orbital:

$$
\mathrm{N}(1 / 2)=1, \Rightarrow \mathrm{r}_{\mathrm{o}}^{*}(1 / 2)=\mathrm{e} /\left(\sigma_{\mathrm{e}} \cdot 2 \pi \cdot 1_{\sigma}\right)=\mathrm{r}_{\mathrm{o}} / 2
$$

For other atoms, with bigger mass, the transition on subfundamental level: $(n=1) \rightarrow\left(n^{\prime}=1 / 2\right)$ results as possible by stimulated electronic transition, according to the model, (by laser excitation with: $\mathrm{h} v=\mathrm{E}_{1}-\mathrm{E}_{1 / 2}$, resulting possible also the producing of "mascons" (concentrated mass, resulted by the atomic radius decreasing and stronger inter-atomic forces). Itresults also that- by this possibility, it may be generated also a stimulated $\mathrm{K}^{1} / 2$-electronic capture to some atoms (such as Am), after electronic induced transition on subfundamental energetic level $(n=1 / 2)$, according to the model, by a nuclear reaction in the form: ${ }^{1}$

$$
\mathrm{p}^{+}+\mathrm{e}^{-} \rightarrow \mathrm{n}^{0}+v_{\mathrm{e}} ;_{\mathrm{Z}} \mathrm{N}^{\mathrm{A}}+\mathrm{e}^{-} \rightarrow_{(\mathrm{Z}-1)} \mathrm{M}^{\mathrm{A}}
$$

\section{The Kervran effect}

An unexplained phenomenon by the usual physics was evidenced in 1962 by Louis Kervran and it refers to the phenomenon of atomic transmutations at low un-radiative energy, produced for stable isotopes by a biological organism. Her assumption is based on previous observations of french chemist Vauquelin, who observed that a hen nourished with oats and water, produced of five times more calcium than the consumed quantity. Prout observed also that an egg of one day has of four times more calcium than the fecundated egg. Reseaches concerning the variation of calcium indicated similar disproportions during the germination of the oats seeds (Von Herzeele, 1875-1883) and barley, (Long, 1970).

During 1875 and 1883, von Herzeele conducted 500 analytical experiments which checked the growing of plants in controlled medium and he concluded that the plants can produce nuclear transmutations of some chemical elements. Similar researches were done by Baranger from the Polytechnic School in Paris, 1947, who analyzed the content of $\mathrm{Ca}, \mathrm{K}$, and $\mathrm{P}$ in plants. His researches showed that the un-germinated seeds or germinated in distilled water doesn't reveal a variable content of $\mathrm{K}$, but the seeds treated with $\mathrm{CaCl}_{2}$ has an increased quantity of $\mathrm{P}$, unexplained by the plants biology, and a growth of $10 \%$ of K. ${ }^{8}$ After Kervran, the living organisms can produce, in certain conditions, by bio-geochemical reactions, also nuclear reactions of elements as: $\mathrm{C}, \mathrm{N}, \mathrm{O}, \mathrm{Si}, \mathrm{Na}, \mathrm{K}, \mathrm{Ca}, \mathrm{P}, \mathrm{S}, \mathrm{Kl}$, by specific enzimes (transmutantenzimes) located intra-mitochondrial. ${ }^{9}$ For explain the effect of biological transmutations of chemical elements, L. Kervran considered a new nuclear model, as cluster of alpha particles with two types of links: hard and weak, considering that the weak links can be split enzymatically, so that an atomic nucleus can be divided in two nuclei by biological way, with the mitochondrial energy, the produced energy being a part of the total energy of the body. We can observe that this nuclear model is in partial concordance with the equation (2) of nuclear potential periodical decreasing, resulted in CGT as consequence of the deuteronic self-resonance at the level of a heavier nucleus.

Kervran considered that $\mathrm{Si}$ is a "bio-consumed" element and $\mathrm{Ca}$ is a bio-produced element, being known that it is possible to reduce the lack of $\mathrm{Ca}$ in the human body and in the animal body (cow, pig) through the administration of $\mathrm{Mg}$ and $\mathrm{Si}$ (under different formsorganic or anorganic). According to Kervran, the specific reactions are:

$$
{ }^{24} \mathrm{Mg}+{ }^{16} \mathrm{O} \rightarrow{ }^{40} \mathrm{Ca} ;{ }^{28} \mathrm{Si}+{ }^{12} \mathrm{C} \rightarrow{ }^{40} \mathrm{Ca}
$$


It is considered also that in the process of thermolysis and of ATP modification, in the human body, by electric excitations are produced the following reactions:

$$
{ }^{39} \mathrm{~K}+{ }^{1} \mathrm{H} \rightarrow{ }^{40} \mathrm{Ca} ;{ }^{23} \mathrm{Na}+{ }^{16} \mathrm{O} \rightarrow{ }^{39} \mathrm{~K},
$$

De Beauregard proposed to explain the biological transmutation of potassium by the known reaction:

$$
{ }^{39} \mathrm{~K} \rightarrow{ }^{39} \mathrm{Ca}+\mathrm{e}^{-}+\bar{v}_{\mathrm{e}} ;\left(v_{\mathrm{e}}-\text { antineutrino }\right)
$$

G. Oshava and M. Torii showed in $1964^{10}$ that after an electrical discharge of $60 \mathrm{~W}$ and 30 minutes, in a vacuum tube of $20 \mathrm{~cm}$ containing $2.3 \mathrm{mg}$ of $\mathrm{Na}$, by introducing of $\mathrm{O}$ in the tube, after the stopping of electrical discharges is produced a cold fusionbetween nuclei of $\mathrm{Na}$ and $\mathrm{O}$, producing $\mathrm{K}$ as in (13') reaction which explains the adjustment of $\mathrm{Na} / \mathrm{K}$ balance to cell level, according to the Kervran effect. Also this observation is in concordance with the eqn. (2) of CGT, indicating a nuclear potential's periodical decreasing by nucleus' vibration. L. Kervran and Komaki showed after many years of observations that the human and the animal bodies consumes continuously $\mathrm{Na}$ and eliminates continuously $\mathrm{K},{ }^{9}$ but the $\mathrm{Na} / \mathrm{K}$ balance remains constant, with or without $\mathrm{K}$ consuming. Experimental researches in the field of biological nuclear transmutations producing, have been realized by Panos T. Pappas from the Physics Department of Pirraeus Technological Institute, which evidenced the role of the cell membrane potential, ${ }^{11}$ sustaining the phenomenon of $\mathrm{S}$ transmutation in potassium inside the biological cell, during the processes of active (Na-K) pump of ions in the presence of oxygen, according to the reaction:

$$
{ }_{11} \mathrm{Na}^{23}+(\text { electric excit. })+{ }_{8} \mathrm{O}^{16} \rightarrow_{19} \mathrm{~K}^{39}+\text { Energy }
$$

which was considered in the base of G. Oshava and M.Torii researches (1964) and those of Hodkin and Keynes (1955).

During 1989-1999 the researches made by P. Pappas showed that the concentration of $\mathrm{K}$ increases in the blood of the bodies subjected to magnetic pulses of short time, which generates induced electricity representing a fraction from the value of transmembranar potential gradient which is of about $10 \mathrm{MV} / \mathrm{m}$, to a power level corresponding to the thermal level of electrotherapy. ${ }^{12}$ It is considered that a great number of functional biological and medical mechanisms could be better understood through the known mechanisms of osmosis correlated with the reaction (16) of biological nuclear transmutation, implying also the energy of ATP transforming, at the cell level, according to the reaction:

$$
\begin{aligned}
{ }_{11} \mathrm{Na}^{23}+{ }_{8} \mathrm{O}^{16} & +(\text { electricexcit. })+(\mathrm{ATP}-\text { aze' energy }) \rightarrow \\
& \rightarrow{ }_{19} \mathrm{~K}^{39}+\text { BioEnergy }
\end{aligned}
$$

and with its reverse:

$$
{ }_{19} \mathrm{~K}^{39}+(\text { electricexcit. })={ }_{11} \mathrm{Na}^{23}+{ }_{8} \mathrm{O}^{16}
$$

with the energy of an electric current

There are also other serious studies concerning the Kervran effect and some patented inventions, for example-based on researches of Vladimir I. Vysotsky, Alla A. Kornilova and Igor I. Samajlenko (patent: RU2052223/10 January 2006).

\section{A possible explanation of the phenomenon}

The explaining of these nuclear reactions of cold transmutations through the nuclearmolecule model typeand through the vortexialmodel of atom, proposed in CGT, supposes the hypothesis that the energy of intra-mitochondrial transformations (of some specific transmuting enzymes) or some electrical impulses delivered in shocks, increases the vibrating state of the atomic components (electrons and nuclei) and favors the nuclear fusion or fissionbythe periodical decreasing of the nuclear potential in accordance with the equation (2) of deuteronic self-resonance $\left(\mathrm{CGT}^{1,2}\right)$ induced in this case by the nucleus' vibration, the nuclear fusion reactions of the Kervran's effect resulting and as a consequence of electronic transition on subfundamental energetic level (of $n=1 / 2$ ), induced by the sinergonoquantonic vortex of the nuclear magnetic moment and by the energy of the ATP transformation or/and by electric impulses or by nuclear magnetic resonance, the nuclear charge screening produced by the e-charge of this electronic $n=1 / 2$ level favoring the nuclear fusion as in the case of reaction (17), by atoms coupling with collinear magnetic momenta. The previous hypothesis is in accordance with the Kervran's conclusions looking the role of intra-mitochondrialenergy resulted by the ATP transformation and with the Pappas explicative model based on the role of the cell membranepotential in the phenomenon's producing. Also, there are some experiments of cold nuclear fusion obtained with relative low electric potential, such as:

$$
\mathrm{O}+\mathrm{H} \rightarrow \mathrm{N} \text {;(Th. Moray, 1927); }
$$

Light $\rightarrow \mathrm{Be} 8$; (Cockcroft is Walton, 1932, 125V).

An US Patent: 1427109, was granted in 1965 to M.Y.Sakurazawa for the invention: "Manufacture of special steels by means of low energy transmutations", and implies also a transformation of low energy and temperature, of the type:

$$
2 \mathrm{C}+20 \rightarrow 2 \mathrm{Si}+2\left({ }^{56} \mathrm{Ni}_{28}\right) \rightarrow\left\{{ }^{56} \mathrm{Fe} ;{ }^{58} \mathrm{Ni}\right.
$$

produced by an electric discharge at $80 \mathrm{~V}$ and 15A, between carbon electrodes, in a chamber with pure oxygen or air.

Also are known the experiments of Walter Russell resumed from Tim Binder et al. ${ }^{13}$ for the production of fluorine from pure water vapor, using a complex arrangement of electro-magnetic fields. It is known also the effect Barker-Keller of nuclear transmutation accelerating by electric discharge (US 5.076.971-Apparatus and method for decontaminating radioactive materials by stimulating the atomic system of radioactive materials), the rate of the radioactive decay of the materials being greatly accelerated at potential differences in the range of 50 kilovolts to 500 kilovolts, applied for a period of at least 30 minutes. Looking the nuclear transmutation produced by nuclear fission biologically induced, considered by the Kervran effect, we may suppose that the increasing of nucleus vibration energy by chemobiological reactions, determines the reducing of the quantonic vortex $\Gamma_{\mu}{ }^{\mathrm{N}}$ of the nuclear magnetic moment and the increasing vibration energy of some weakly linked nucleons, determining- according to the quasi-crystal nuclear model of CGT, the local decreasing of the bonding energy of vibrated nucleons, conform to eq. (2) and thereafter - the nuclear fission of the nucleus in two stable nuclei, by nucleonic self-resonance. The previous explanation is relative equivalent with those of Kervran, considering a nuclear model of alpha particles with two types of links: hard links and weak links. 
Relative to the reaction (14): ${ }^{39} \mathrm{~K}+{ }^{1} \mathrm{H} \rightarrow{ }^{40} \mathrm{Ca}$,

it is observed that -even it can explain the calcium generating by $\mathrm{K}$, it seems un-plausible as biological cold fusion reaction, because the Coulombian nuclear barrier. For example, for deuteron the Coulomb barrier is: $\mathrm{B}_{\mathrm{C}}=\mathrm{zZe}^{2} /\left({ }^{3} \sqrt{\mathrm{A}}\right)=0.8 \mathrm{MeV}$ - too high for the possibility to occurring inside a biological cell, even in the context of the proton's charge screening by the hydrino atom's electron. However, according to the Quantum Mechanics, the charged particle $m_{p}$ can pass over the potential barrier of high $\mathrm{U}_{0}$ and with a by a "tunneling effect", with a probability $\mathrm{P}_{\mathrm{t}}$ given by the relation:

$$
P_{t}=\exp \left(-\frac{2}{\hbar} a \int_{r_{1}}^{r_{2}} \sqrt{2 m_{p}\left(U_{0}-T\right)} d\right)
$$

in which $\mathrm{T}$ is the particle's kinetic energy along r-direction. But the classical law of energy conservation impose :

$\mathrm{T} \geq \mathrm{U}_{0}$. Of classical point of view, it seems that the tunneling probability $\mathrm{P}_{\mathrm{t}}$ indicates the probability of the $\mathrm{U}_{0}$-potential barrier' decreasing during the interaction of particles $m_{p}(z)$ and $M_{p}(Z)$. This imply the probabilistic reducing of the Z- or $\mathrm{Z}$-charge of the particle $\mathrm{m}_{\mathrm{p}}$ orM $\mathrm{p}_{\mathrm{p}}$. It may be supposed- for example, an oscillating z-charge of the proton, as in the Leo Sapogin's theory ${ }^{14}$ which considers a periodical variable charge as effect of the associated wave function of the particle. But for sustain also phenomenologically this hypothesis, is necessary to known the e-charge's generation. According to the prequantum multi-vortexial model of nucleon of CGT, the cold electron is of barrel-like form and the e-charge is given by a flux of vectorial photons ('vectons'-in CGT) resulted by vortexial conversion from pseudo-scalar photons of the quantum vacuum and spread quasiradially by the $\Gamma_{\mu}$-vortex of the particle's magnetic moment. Because the precession movement, the vectons' spreading seems spheric- symmetric, even if they are emitted with the impulse quasi-rectangular on the particle's spin. When the m-particle pass through the quantum

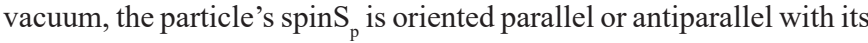
impulse $p_{p}=m v$, as in the case of beta transformation of the neutron. If this alignment is proportional with the particle's impulse $p$, will result an anisotropy of the particle's e-charge-considered contained by its surface, because the spreading of a lower flux of 'vectons' of electric interaction in the polar zones, the $\mathrm{P}_{\mathrm{t}}$ - probability of potential barrier tunneling being in this case explainable in concordance with the energy conservation law. It results also that- of classical point of view, the wavelength $\lambda_{\mathrm{p}}=\mathrm{h} / \mathrm{m} \cdot \mathrm{v}$ associated to a moving particle and the associated wave-function $\Psi(r)$ may describe the particle's spin precession, whose frequency may be proportional with its impulse and its kinetic energy, i.e: $v_{\mathrm{p}}=\mathrm{v} / \lambda_{\mathrm{p}}=\mathrm{mv}^{2} / \mathrm{h}$. When $v_{\mathrm{p}}$ increases, the precession oscillation amplitude decreases and the parallelism between the spin $\mathrm{S}_{\mathrm{p}}$ and the impulse $\mathrm{p}_{\mathrm{p}}$ increases.

We may suppose also aperiodical decrease of the nucleus' charge, $\mathrm{Q}=\mathrm{Ze}$, generated by a periodical diminishing of the protons' vortexiality during a monopolar giant resonance, i.e-the nucleus' volume pulsating as consequence of the deuteronic self-resonnance generated by the nuclear potential periodically diminishing at the nucleons' impact or at the nucleus' vibration, according to eqn. (2). The way in which the nucleus vibration may generates periodically Q-charge decreasing results by the multi-vortexial nucleon model of CGT by the induced intrinsic vibration of the electronic super-dense centroids inside the nucleon's volume, at its vibration with high frequency, with intrinsic vortexial (vexonic) sub-structures destroying, (with the transforming of their dynamical quantonic pressure into static pressure), phenomenon which generates the absorption of electric interaction vectorial photons (vectons), by vibration with the energy $\Delta \varepsilon_{v}$ by a reaction of type: ${ }^{1}$

$$
\mathrm{n} . \varepsilon_{\mathrm{i}}+\mathrm{m}_{\mathrm{p}} \mathrm{c}^{2}+\Delta \varepsilon_{v} \rightarrow \mathrm{m}_{\mathrm{p}} \mathrm{c}^{2}+\varepsilon_{\mathrm{w}} ; \varepsilon_{\mathrm{w}}=\mathrm{n} . \varepsilon_{\mathrm{i}},\left(\mathrm{n}^{3} \geq 10\right) ; \varepsilon_{\mathrm{i}}=\mathrm{h} v_{\mathrm{i}} ; \varepsilon_{\mathrm{w}}=\mathrm{h} v_{\mathrm{v}} ; \Delta \varepsilon_{v} \geq^{3} \varepsilon_{\mathrm{w}} / \mathrm{K} ; v_{\mathrm{v}}=10^{15}-10^{20} \mathrm{~Hz}
$$

where: $\varepsilon_{i} ; \varepsilon_{w}$ - the energy of the captured photons and, respectively, of thee mitted scalar quantum generated by electromagnetic vibration with an energy $\Delta \varepsilon_{v}$ per emitted scalar quantum and $K$ is a constant which can be of over-unity value-according to some experiments, without contradiction with the energy conservation law. The nuclear $\mathrm{Q}(Z)$-charge's variation at the atom's sinusoidal vibration with amplitude $\mathrm{A}: \mathrm{x}=\mathrm{A} \cdot \sin (\omega \cdot \mathrm{t}),(\omega=2 \pi v)$, results of the form: $\mathrm{Q}=\mathrm{Q}_{0}-\Delta \mathrm{Q}$, with $\Delta \mathrm{Q} \in_{v}$ :

$$
\begin{gathered}
U_{t} \omega(r, \omega)=\left(U_{0}(r)-\Delta U(r, \omega)\right)=U_{0}\left(1-\Delta Q / Q_{0}\right) \\
\Delta U=\mathrm{k}_{v} \in_{v} ; \epsilon_{v}=1 / 2 \mathrm{~m}_{\mathrm{N}} \mathrm{v}^{2}=1 / 2 \mathrm{~m}_{\mathrm{N}}\left(\omega \cdot \mathrm{A}^{2} \cos ^{2}(\omega \cdot \theta)\right.
\end{gathered}
$$

( $v$ - the vibration' frequency), resulting that:

$$
\mathrm{Q}=\mathrm{Q}_{0}\left(1-\mathrm{k}_{v} \in_{v} / \epsilon_{v}{ }^{0}\right)=\mathrm{Q}_{0}\left(1-\mathrm{K}_{v} \epsilon_{v}\right) ;\left(\mathrm{Q}_{0}=\mathrm{e} \cdot \mathrm{Z}\right)
$$

with: $\left(\mathrm{k}_{v}<1 ; \varepsilon_{v} \leq \varepsilon_{v}{ }^{0}\right) ; \mathrm{k}_{v} ; \mathrm{K}_{v}$-proportionality constants.

The previous hypothesis cannot explain the dependency of the potential barrier tunneling probability on the incident particle's impulse, but contributes to the explaining of the observed reaction of cold nuclear fusion, (which generally implies a nuclear excitation energy).

\section{Other implications of the theoretical model}

However, the previous possibility may sustain in CGT a technical possibility: those of the realizing of a weight decreasing for a metamaterial structured as metallic sandwich of 1-5 mm with an upper diamagnetic layer (as $\mathrm{Bi}$ ), a median dense layer ( $\mathrm{Pb}$ or 'mascon'), an intermediate strong magnetic layer and an inferior metallic layer strongly dopped with nuclei with giant resonance: $\mathrm{U}, \mathrm{Zr}, \mathrm{Pb}$ excited with gamma rays with energy $\mathrm{E}_{\gamma} \rightarrow 1 \mathrm{MeV}$ or even with microwave of nuclear magnetic resonance, $(v \rightarrow 1 \mathrm{GHz})$, which-by the induced deuteronic(self)resonance, will increase the local static quantonic pressure by partially destroying of the internal vortexiality (by the vibration of the electronic superdense kernels inside the vibrated nucleonic volume), which will generate a quantonic pressure gradient corresponding to a quantic pseudo-antigravitic (levitation) force, (an inverse Casimir effect), acting over the rest part of the metallic sandwich according to CGT. ${ }^{1,15}$ The median magnetic layer may generate also a quantum repelling (levitating) force over the diamagnetic layer, (which is repelled by its magnetic field), without the generating of a reactive force. Another theoretic implication of the presented hypothesis regarding the nuclear cold fusion is in the explaining of the metallic elements spreading inside the earth's surface. 
In this sense, it is known the oppinion that the majority of the atomic elements are synthesized inside the stars, at very high temperature, for example, in a supernova explosions, in conditions in which the kinetic energy of the lightest nuclei $(\mathrm{H}, \mathrm{D}, \mathrm{He})$ exceeds the repulsive coulombian potential and favores their fusion. But in this case the protoplanetary nebula which formed also the Earth (according to the Kant-Laplace theory) should contain homogenously spreading elements. So, in consequence, the orogenesis theories cannot explain enough clear (causally) the localized spreading of metallic ores and of other deposits of telluric chemical elements. Also, it is known that the asteroids are heterogenous as chemical composition, being classified into three groups: C-type (carbonaceous) asteroids, which comprise about $75 \%$ of the total and are similar in composition to carbonaceous chondrites, the most common class of meteorite; S-types (of stone) which comprise about $15 \%$ and contain various silicate compounds and a certain amount of nickel and/or iron and of M-types (metallic), with a predominantly iron content and thus a link with iron meteorites. Contrary to the expectations of the nebula hypothesis, it is now clear that they do not form an orderly compositional gradient across the main belt between Mars and Jupiter but are jumbled together, especially the smaller ones. An example is the asteroid Psyche 16, found between Mars and Jupiter, which is made of solid metal: gold, platinum, iron and nickel.

Also, because the heat generated by radioactivity seems to have been insufficient to cause large-scale melting, it is supposed that the asteroid Vesta, (530 km in diameter), which is an achrondritic meteorite,derived from the explosion of a still larger molten body and its internal melting caused light minerals (silicates) to float towards the surface, its centre remaining with heavy minerals, rich in iron. It exist two basic hypothetical possibilities to explain the localized spreading of telluric elements: -either i) the Earth ,inherited' neomogenities in the chemical elements' spreading from the protoplanetary nebula (suggesting the cold forming of heavier nuclei as quasi-crystalline clusters of protons and neutrons- possibility resulted in CGT by a 'dynamide' model of neutron, with degenerate electron(s) inside the nucleon's volume, ${ }^{1,2}$ ), -or: ii) the heavier elements were formed at least partially inside the Earth, from lighter elements, at high temperatures and pressures (but lower than those specific to stars) and in conditions of intense electric discharges or intense vibration, particularly- during the orogenesis processes, as in the case of reaction (17) of ,cold fusion', for example.

The chemical composition of Jupiter, whose atmosphere is composed fromhydrogen and helium in a proportion close to the theoretical composition of the primordial solar nebula, its interior containing roughly $71 \%$ hydrogen, $24 \%$ helium, and $5 \%$ other denser elements, ${ }^{16}$ suggests that is more plausible the second explanatory variant ii) or a mixt variant, i.e: -iii) the forming of heavier elements inside the planet, by 'cold' fusion of a solid kernel resulted from cosmic dust formed by heavier nuclei generated in a cold genesis process (at $\mathrm{T} \rightarrow 0 \mathrm{~K}, \mathrm{CGT}^{17}$ ) with $\mathrm{H}-$ and He-nuclei, this process being in concordance with the fact that-in the case of Psyche 16 asteroid, the difference between the Au-nucleus and the Pt-nucleus is a proton and the difference between a $\mathrm{Fe}$-nucleus and a Ni-nucleus is a He3-or a He4-particle.

It is believed that the cosmic dust results from chemical elements generated inside the stars, especially- inside the supernovae and expelled in the outer space, an important argument being the fact that the emission at 11.5 micrometres indicates the presence of silicon carbide dust in cool evolved carbon-rich giant stars. ${ }^{18}$ The hypothesis of cosmic dust forming from heavier nuclei generated at cold, ${ }^{17}$ especially in the gravitational field of a 'black hole' or a magnetar-type star, from neutrons "escaped" from a neutronic star's surface, is in concordance with some reports that showed some evidences that the cosmic dust is formed near a supermassive black hole. ${ }^{19}$ This hypothesis is sustained also by the fact that-although the most abundant elements in the Sun are hydrogen and helium, a total of 60 elements were abundant in both meteorites and solar photosphere, suggesting that these elements were probably abundant also in the protosolar nebula. The research of Trevor Ireland and coauthors ${ }^{20}$ compared the composition of the Earth's core and those of the primordial mantle of the planet, which can be calculated using a combination of mathematical models, seismic data and rock samples. They found that the Earth was "devolatilized" over time, and this was an "inherent process" as the Solar System formed,concluding that the exoplanets are almost certainly devolatilized pieces of star nebula, This conclusion is in concordance with the possibility to explain the Titius-Bode relation of planet-Sun distances by the Kant-Laplace theory of the solar system's forming. ${ }^{19}$

Another astrophysical theoretical implication of the vortexial model of atom and of the multi-vortexial model of nucleon proposed in CGT is related to the process of matter $\rightarrow$ energy conversion resulted at the surface of a massive or super-massive 'black hole' type star because the strong gravity at the BH's surface but also because the nuclear force and the diminishing of the repulsive character of the impenetrable nucleonic volume' field at $\mathrm{T} \rightarrow 0 \mathrm{~K}$ (resulted according to CGT $^{21}$ ). According to CGT, the releasing of the nucleon's energy is realized- after the atom's implosion with the capturing of the atomic electrons by the nuclei and the transforming of the nuclear protons into neutrons, by the nuclear matter partial transforming into quark matter, the rest part of neutrons being destroyed with the releasing of their intrinsic vortexial energy $\mathrm{m}_{\mathrm{n}} \mathrm{c}^{2}$ into etherono-quantonic fluxes which generates periodically an anti-gravitational (pseudo)charge of the BH's, according to CGT, ${ }^{2}$ being explained in this way the cause of Universe expansion but also the mechanism of gravitational waves emission and the astrophysical observation of a star which is moving away from a black hole, even if this $\mathrm{BH}$ could not generate its acceleration, (the star PG1610+062, ${ }^{22}$ ). According to CGT, because the etheronic flux of 'dark energy' released by a periodical matter $\rightarrow$ energy conversion process is much intense than the mean gravitonic flux of etherons coming toward the 'black hole' star, (which generates the gravitation force- according to a Fatio-LeSage model ${ }^{1,2}$ ), the mean total effect can be a carrying off, generated by a repulsion force $\mathrm{F}_{\mathrm{r}}$ of high value acting in short periods of time $\Delta \mathrm{t}_{1}$ followed by a less intense attraction force $\mathrm{F}_{\mathrm{a}}$ acting in longer period of times, $\Delta \mathrm{t}_{2}$, i.e:

$$
\mathrm{F}_{\mathrm{r}} \Delta \mathrm{t}_{1}>\mathrm{F}_{\mathrm{a}} \Delta \mathrm{t}_{2}
$$

It is explained in this way also an apparently strange cosmic phenomenon: the recently discovery of a star:S5-HVS1, which is to about 29,000 light-years from Earth and pass through the Crane constellation in the southern sky because was expelledfrom the center of our galaxy (in whichis a super-massive black hole known as Sagittarius A*, with the mass of four million solar masses)at the speed of four million miles/hour- of ten times higher than the mean speed of the galactic stars. ${ }^{23}$ The phenomenon is explained the by the 'Hills mechanism', by which the supermassive black hole in the center of the Milky Way can eject stars when a binary star system is disrupted by a supermassive black hole: the tidal forces from the black hole cause one of the stars to be captured by it, falling into an orbit around it, the other star being jettisoned away from it at very high speeds. According to CGT, the phenomenon is explained by a periodically 
anti-gravitic pseudo-charge of the super-massive BH "Sagittarius A*, which may contribute also to the galaxy's stability (to its dynamic equilibrium). Also, according to CGT, if the strong gravitic field of the $\mathrm{BH}$ converts partially the $\mathrm{H}-$ and He-nuclei of another star into neutrons, the expelling of these neutrons during the matter $\rightarrow$ energy conversion and the BH's antigravitic pseudo-charge forming gives the neutronic mass for heavier nuclei cold forming ${ }^{17}$ and for cosmic dust cold forming- in concordance with the astrophysical observations showing that the cosmic dust is formed near a supermassive black hole. ${ }^{19}$

Another astrophysical phenomenon which may be explained by the conclusion of the periodically generating of ablack hole' anti-gravitic pseudo-chargeis the observing of a supernova called iPTF14hls, of II-P type, with a core collapsed into a neutron star, which after a first explosion in 1954 exploded again after 60 years, after 600 days of observation its light decreasing and increasing in intensity repeatedly at least of five times. ${ }^{24}$ The phenomenon could not be explained as pair-instability supernova effect- generated when the production of free electrons and positrons in the collision between atomic nuclei and energetic gamma rays, temporarily reduces the internal pressure of a super massive stars core. According to CGT, it is possible the generating of the effect by matter $\rightarrow$ energy conversion at the surface of super nova's core of 'black hole' type, with periodically generating of an antigravitic pseudo-charge, $(\mathrm{PBH})$ phenomenon coherently explained in CGT. ${ }^{2}$ Also, the gammonic un-destroyed pairs $\left(\mathrm{e}^{-} \mathrm{e}^{+}\right)^{*}$ of nucleonic degenerate electrons emitted as hard gamma-rays, of $1 \mathrm{MeV}$, by their conversion into negatron-positron pairs in a strong electric or magnetic field like those of a charged 'black hole' or of a magnetar, may explain the mysterious presence of antimatter in the middle of the Milky Way- phenomenon considered initially as caused by particular type of supernova, ${ }^{25}$ hypothesis which cannot explain how is generated a quantity of 15 billion tons of positrons per second. Another possibility predicted in CGT which may explain the positrons production in the field of a massive black hole or of a magnetar is the genesis of $\left(\mathrm{e}^{-} \mathrm{e}^{+}\right)$pairs from the quantum vacuum, at very low temperatures $\mathrm{T} \rightarrow 0 \mathrm{~K}$ but in an enough intense magnetic field. ${ }^{26}$

\section{Conclusion}

In the paper is presented a vortexial pre-quantum model of atom, based on a vortexial nature of the electron' and proton' magnetic moment, resulted in a Cold Genesis Theory as etherono-quantonic vortex: $\Gamma_{\mu}^{*}(\mathrm{r})=\Gamma_{\mu}\left(\mathrm{r}_{\mu}{ }^{\prime}\right)+\Gamma_{\mathrm{B}}\left(\mathrm{r}>\mathrm{r}_{\mu}{ }^{\prime}\right)$, of, heavy' tachyonic etherons $\left(\mathrm{m}_{\mathrm{s}} \approx 10^{-60} \mathrm{~kg} ; \mathrm{w}>\mathrm{c}\right)$ - generating the magnetic potential $\mathbf{A}$, and of quantons $\left(\mathrm{m}_{\mathrm{h}}=\mathrm{h} \cdot 1 / \mathrm{c}^{2}=7.37 \times 10^{-51} \mathrm{~kg}\right)$ - generating vortex-tubes $\xi_{\mathbf{B}}$ that materializes the $\mathbf{B}$-field lines of the magnetic induction, the proton's magnetic moment resulting by a degenerate Compton radius. The model may explain the 'hydrino' atom, with $n=1 / 2$, the tachyonic speed of the electronic neutrino and the Kervran effect of biological nuclear transmutations. By the multi-vortexial model of nucleon resulted in CGT, are explained also some astrophysical observation which sustains the CGT's hypothesis of pulsatile antigravitic pseudocharge and gravitational waves generating at the surface of a 'black hole type star by matter-energy conversion.

\section{Acknowledgments}

None.

\section{Conflicts of interest}

Author declares that there are no conflicts of interest.

\section{Funding}

None.

\section{References}

1. Arghirescu M. The material structures genesis and field effects. $2^{\text {nd }}$ ed. MatrixROM, Bucharest. 2006.

2. Arghirescu M. The Cold Genesis of Matter and Fields. $2^{\text {nd }}$ ed. Science PG. 2015.

3. Arghirescu M. A Quasi-Unitary Pre-Quantum Theory of Particles and Fields and Some Theoretical Implications. IJHEP. 2015;7(1)80-103.

4. A Săndulescu. Alpha reduced widths on super fluid model. Nucl Physics. 1963;48:345-351.

5. Arghirescu M. The nuclear force explaining by a bag model resulted from a vortexial, cold genesis model of nucleon. Phys Astron Int J. 2018;2(4):349-358.

6. The OPERA Collaboraton. Measurement of the neutrino velocity with the OPERA detector in the CNGS beam. ar Xiv: 1109.4897v1.

7. SE Harris, LV Hau, Nonlinear Optics at Low Light Levels. Phys Rev Lett. 1999;82:4611.

8. A Brunel-Tourcoin. Traite' practique de chemie des plants. Paris. 1948.

9. LC Kervran. Transmutation biologiqueset Physique Moderne. $1^{\text {st }}$ ed. In: Maloine SA editor. Paris. 1982.

10. Roberto Monti. Fusione Fredda e relativita Einsteiniana: Statodell'Arte, Reprint, Societa Editrice Andromeda, Bologna. 1996;9:71.

11. PT Pappas. PAP-IMI Cases Reports, (1990-1998), Electrically Induced Nuclear Fusion. Journal of New Energy. 1998;3(1).

12. Meyer S. Walter fuel cell. Technical brief. 1991.

13. Leo Sapogin. The Unitary Quantum Theory and New Sources of Energy. $1^{\text {st }}$ ed. Science PG, 2015.

14. Marius A. The Generation of Propulsion Force by the Quantum Energy: The "E-M Drive" Case. Open Access Library Journal. 2017;4:e3363.

15. Kunde VG, FM Flasar,DE Jennings, et al. Jupiter's Atmospheric Composition from the Cassini Thermal Infrared Spectroscopy Experiment. Science. 2004;305(5690):1582-86.

16. M Arghirescu. The Explaining of "Magic" Nuclear Numbers by a QuasiCrystalline Nuclear Model, of Possible Cold Genesis. Journal of Particle Physics. 2020;4(1).

17. Humphreys RM, Strecker DW, Ney EP. Spectroscopic and Photometric Observations of M Supergiants in Carina. Astrophysical Journal. 1972;172:75.

18. Markwick-Kemper F, Gallagher SC, Hines DC, et al. Dust in the Wind: Crystalline Silicates, Corundum, and Periclase in PG 2112+059. Astrophysical Journal. 2007;668(2):L107-L110.

19. HS Wang, CH Lineweaver, TR Ireland. The elemental abundances (with uncertainties) of the most Earth-like planet. Icarus. 2018;299:460-474.

20. Marius A. The nuclear force as main cause of the black hole's forming and increasing, in accordance with a multi-vortexial model of particle, of cold genesis. Global Journ of Phys. 2019;9(2):853-859.

21. Brandon Specktor. This Ridiculously Speedy Star Might Be Running Away from a Rare, Unproven Type of Black Hole. Science \& Astronomy. $2019 ; 13(9)$

22. SE Koposov, D Boubert, Ting S, et al. The Great Escape: Discovery of a nearby $1700 \mathrm{~km} / \mathrm{s}$ star ejected from the Milky Way by Sgr A*. arXiv:1907.11725v2. 
23. Michelle S. This Star Exploded, Survived, and Then Exploded Again After 60 Years. Sci Alert. 2017.

24. Bob Berman. Weird Object: Milky Way Antimatter Fountain. Astronomy Rev. 2015;8.
25. M Arghirescu. The Possibility of Particles Forming from a Bose-Einstein Condensate, in an Intense Magnetic or Gravitational Field. Int J of High En Phys. 2018;5(1):55-62. 